\title{
Induction of insulin-like growth factor 2 expression in a mesenchymal cell line co-cultured with an ameloblast cell line
}

\author{
Asako Matsumoto • Hidemitsu Harada • \\ Masahiro Saito • Akiyoshi Taniguchi
}

Received: 30 May 2011 / Accepted: 9 September 2011 / Published online: 30 September 2011 / Editor: T. Okamoto

(C) The Society for In Vitro Biology 2011

\begin{abstract}
Various growth factors have been implicated in the regulation of cell proliferation and differentiation during tooth development. It has been unclear if insulin-like growth factors (IGFs) participate in the epithelium-mesenchyme interactions of tooth development. We previously produced threedimensional sandwich co-culture systems (SW) containing a collagen membrane that induce the differentiation of epithelial cells. In the present study, we used the SW system to analyze the expression of IGFs and IGFRs. We demonstrate that IGF2 expression in mesenchymal cells was increased by SW. IGF1R transduces a signal; however, IGF2R does not
\end{abstract}

A. Matsumoto $\cdot$ A. Taniguchi $(\bowtie)$

Cell-Materials Interaction Group, Biomaterials Unit,

National Institute for Materials Science,

1-1 Namiki,

Tsukuba, Ibaraki 305-0044, Japan

e-mail: taniguchi.akiyoshi@nims.go.jp

A. Matsumoto $\cdot$ A. Taniguchi

Biomaterials and Tissue Engineering,

Graduate School of Comprehensive Human Science,

University of Tsukuba,

Tsukuba, Ibaraki 305-8572, Japan

\section{A. Matsumoto}

Kyokuto Pharmaceutical Industrial Co., Ltd.,

3333-26 Aza-Asayama, Kamitezuna,

Takahagi-shi, Ibaraki 318-0004, Japan

\section{H. Harada}

Department of Oral Structure and Function Biology,

Iwate Medical University,

School of Dentistry, 1-3-27 Chuodori,

Morioka, Iwate 020-8505, Japan

\section{Saito}

Tissue Engineering Research Center,

Tokyo University of Science,

Noda, Chiba 278-8510, Japan transduce a signal. Recombinant IGF2 induces IGF1R and IGF2R expression in epithelial cells. IGF1R expression is increased by SW; however, IGF2R expression did not increase by SW. Thus, IGF2 signaling works effectively in SW. These results suggest that IGF signaling acts through the collagen membrane on the interaction between the epithelium and mesenchyme. In SW, other cytokines may be suppressed to induce IGF2R induction. Our results suggest that IGF2 may play a role in tooth differentiation.

Keywords Tooth development · Co-culture IGF . Ameloblast

\section{Introduction}

Tooth development is a classic example of epitheliummesenchyme interactions, and it provides a useful experimental system for understanding the molecular mechanisms of organogenesis (Thesleff and Sharpe 1997; Jernvall et al. 2000). During tooth development, various growth factors, such as, fibroblast growth factor, transforming growth factor- $\beta$, and insulin-like growth factor (IGF), regulate cell proliferation and differentiation in a paracrine or autocrine fashion (review by Joseph et al. 1996; Thesleff and Mikkola 2002; Thesleff 2003). To investigate the interaction between epithelial and mesenchymal cells, we previously constructed a three-dimensional culture system by using a collagen membrane, which induces the differentiation of epithelial cells (Matsumoto et al. 2011). Our results suggest that soluble factors produced by mesenchymal cells contribute to the induction of epithelial cell differentiation. Epithelial cell differentiation was induced by soluble factors from dental follicle cells. The molecular weight of soluble factors from dental follicle cells should be small to pass 
through the collagen membrane. We focused on IGFs, which are approximately $8 \mathrm{kDa}$. The IGF family is a complex system comprised of two ligands (IGF1 and IGF2), two cell surface receptors (IGF1R and IGF2R), and several IGF-binding proteins (review by Le Roith 2003). The receptors and binding protein adjust the activity of ligands. IGF2 can bind IGF1R and IGF2R. IGF2R does not transduce a signal, but rather, it serves to limit IGF2 bioavailability by targeting IGF2 for degradation (reviewed by Denley et al. 2005).

The IGFs play a role in tooth formation (reviewed by Werner and Katz 2004), and it has been proposed that IGF1 functions as an autocrine/paracrine regulator of tooth development (Joseph et al. 1996). IGF1 and IGF2 are well known for their mitogenic activities; almost all cell types express IGF1R, so IGFs can stimulate growth and differentiation in many tissues (reviewed by Petley et al. 1999). Previously it has been reported that exogenous IGFs induced the transcription and translation of enamel proteins such as amelogenin and ameloblastin (Takahashi et al. 1998).

The mechanism by which IGFs promote enamel and dentine formation is not well understood. To understand the function of IGFs and insulin-like growth factor receptors (IGFRs) on the interaction between epithelial and mesenchymal cells in tooth development, we used the co-culture system we developed. We analyzed the gene expression and protein localization of IGFs and IGFRs. Our results suggest that IGF2 expression was increased and that IGF2R expression was suppressed in the co-culture system; thus, IGF2 signaling works effectively to eventually induce the differentiation of HAT-7 cells.

\section{Materials and Methods}

Cells and cell culture. The HAT-7 cells used in this study were derived from a dental epithelial cell line that originated from the apical bud of a rat incisor (Kawano et al. 2002). The culture medium consisted of Dulbecco's modified Eagle's medium/F-12 (Invitrogen, Carlsbad, CA) supplemented with $10 \%$ fetal bovine serum (FBS) and penicillin (100 units $/ \mathrm{ml}) /$ streptomycin $(100 \mu \mathrm{g} / \mathrm{ml})$. The $\mathrm{BCPb} 8$ cells used were derived from a dental follicle cell line (a cementoblast progenitor) that originated from the follicle tissue of a bovine incisor (Saito et al. 2005). The culture medium was $\alpha$-minimum essential medium (Sigma, St. Louis, MO) supplemented with $10 \%$ FBS, penicillin (100 units/ml)/streptomycin $(100 \mu \mathrm{g} / \mathrm{ml}), 50 \mathrm{mg} / \mathrm{ml}$ ascorbic acid, and $2 \mathrm{mM}$ L-glutamine (Invitrogen). Fao cells (a kind gift from Prof. K. Motojima, Meiji Pharmaceutical University, Tokyo), a subclone of rat hepatoma HIIE cells, were maintained in F-12 Coon's modification medium
(Sigma) supplemented with $10 \%$ fetal bovine serum, $100 \mathrm{U} / \mathrm{ml}$ penicillin, and $100 \mu \mathrm{g} / \mathrm{ml}$ streptomycin. BPAECs were purchased from Cell Applications, Inc. (San Diego, CA) and cultured in EBM medium supplemented with $5 \%$ fetal bovine serum, $12 \mu \mathrm{g} / \mathrm{mL}$ bovine brain extract, $1 \mu \mathrm{g} / \mathrm{mL}$ hydrocortisone, $10 \mathrm{ng} / \mathrm{ml}$ human epidermal growth factor, $50 \mu \mathrm{g} / \mathrm{mL}$ gentamycin, and $50 \mathrm{ng} / \mathrm{mL}$ amphotericin B using the EGM-MV BulletKit (Cambrex Bio Science, Inc., Wakersville, $\mathrm{MD})$. All cultures were maintained in a humidified atmosphere of $5 \% \mathrm{CO}_{2}$ at $37^{\circ} \mathrm{C}$.

Co-culture using a transparent collagen membrane. Previously, we established two different co-culture models, the sandwich co-culture (SW) system and the separate coculture (SC) system, that used the two dental cell lines and a transparent collagen membrane (Koken, Tokyo, Japan). The molecular weight of soluble factors should be small to pass through the collagen membrane. In brief, in the SW system, HAT-7 cells were inoculated into one side of the collagen film in a culture dish, and the $\mathrm{BCPb} 8$ cells were inoculated into the opposite side of the collagen film. In the $\mathrm{SC}$ system, the $\mathrm{BCPb} 8$ cells were first seeded onto a dish, and then the HAT-7 cells were inoculated into one side of the collagen film. The cell number was $1.1 \times 10^{5}$ cells $/ \mathrm{cm}^{2}$. As a further control, HAT-7 cells were also grown in monolayer culture on a collagen film. HAT-7 cells were cultured in the presence or absence of $0.1,1$, or $100 \mathrm{ng} / \mathrm{ml}$ IGF2 recombinant proteins (R\&D Systems) for $24 \mathrm{~h}$.

$R N A$ extraction, $R T-P C R$, and quantitative real-time PCR analysis. The mRNA levels of differentiation-related marker genes were determined by using RT-PCR or quantitative realtime PCR and species-specific primers (Kurosawa et al. 2005). Briefly, total RNA was extracted at various time points by using ISOGEN (Nippon Gene, Tokyo, Japan). Two micrograms of total RNA was reverse-transcribed into cDNA by using the SuperScript first-strand synthesis system (Invitrogen) according to the manufacturer's protocol.

The primer sets used for RT-PCR are listed in Table 1. Primer sets amplifying glyceraldehyde-3-phosphate dehydrogenase (GAPDH) and beta actin were used as controls. RT-PCR was performed with Ex Taq ${ }^{\mathrm{TM}}$ (Takara, Shiga, Japan). For each template cDNA obtained from the in vitro experiment, a PCR reaction was run by using the primers and conditions shown in Table 1. The PCR products were separated by $1.5 \%$ agarose gel electrophoresis and stained with ethidium bromide. Digital images were taken on an ultra violet background.

Real-time PCR was performed by using an ABI PRISM 7000 Sequence Detection System (Applied Biosystems, Foster City, CA). A standard reaction was performed in a 96-well plate. This reaction was composed of $10 \mu \mathrm{l}$ of 
Table 1. Sequence of oligonucleotide primers for RT-PCR

\begin{tabular}{lll}
\hline Target & Forward & Reverse \\
\hline Rat IGF1 & cacatctcttctacctggcac & agctccggaagcaacactcat \\
Rat IGF2 & caaattcgacacctggagaca & ttgctggacatctccgaagag \\
Rat IGF1R & cacatcctgctcatctccaa & ctcacacatgggcttctcct \\
Rat IGF2R & gggaagctgttgattccaaa & gtcagcgtcagagtcatcca \\
Rat GAPDH & gacccettcattgacctcaactaca & ccaggcccctcctgttgt \\
Bovine IGF1 & cagcagtcttccaacccaat & ttgtttcetgcactcctct \\
Bovine IGF2 & gggatcacagcaggaaagtc & ggtgactcttggcctctctg \\
Bovine IGF1R & gtgacgggctacgtgaagat & acggtgaagctgatgaggtc \\
Bovine IGF2R & tcatgtgcaaaccaggtgat & atattcggggaaacccactc \\
Bovine b-actin & ctggaacggtgaaggtg & agtgtacaggaaagccctgactgcct \\
\hline
\end{tabular}

SYBR Premix Ex Taq ${ }^{\text {TM }}$ II (Takara), 10 pmol each of the forward and reverse primers, $1 \mu \mathrm{l}$ of HAT-7 cDNA, and distilled water to a final volume of $20 \mu \mathrm{l}$. The thermocycling conditions were $95^{\circ} \mathrm{C}$ for $30 \mathrm{~s}$, followed by 40 cycles of $95^{\circ} \mathrm{C}$ for $5 \mathrm{~s}$ and $60^{\circ} \mathrm{C}$ for $34 \mathrm{~s}$. Species-specific primers corresponding to a region of low homology between rat and bovine cDNA were designed with Primer Express Software version 2.0 (Applied Biosystems) based on the sequence of the target gene. The data were normalized by using expression of the housekeeping gene GAPDH as an endogenous control in the same reaction as the gene of interest. The primers used in this study were designated as follows: rat IGF1R forward primer

5'-GGCCATCAGGATTGAGAAAAAC-3', rat IGF1R reverse primer 5'-CACCGCATCCAAGATGAGAGA-3', rat IGF2R forward primer 5'-GCTGTTGATTCCAAAAA TAATGCA-3', rat IGF2R reverse primer 5'-CCACACC TGGGATTATCCACAT-3', rat GAPDH forward primer 5'GCCCCCAACACTGAGCAT-3', rat GAPDH reverse primer, 5'-CCAGGCCCCTCCTGTTGT-3', bovine IGF2 forward primer 5'-TGCCTCTACGACCGTGCTT-3', bovine IGF2 reverse primer 5'-GACTGCTTCCAGATGTCATATTGG-3', bovine IGF1 forward primer 5'-TTGTGATTTCTTGAAGC AGGTGAA-3', bovine IGF1 reverse primer 5'-AGCACAG GGCCAGATAGAAGAG-3', bovine b-actin forward primer 5'-TTGTCCCTCAACTTGAGATGTATGA-3', and bovine b-actin reverse primer 5'-CTGCCTCCGCACCTCAAC-3'.

Immunocytochemistry and immunohistochemistry. The surfaces of the cultured cells were washed three times with phosphate-buffered saline (PBS), and the cells were fixed in $10 \%$ formalin for $10 \mathrm{~min}$. After permeabilization with $0.1 \%$ Triton X-100 in PBS for $5 \mathrm{~min}$, the cells were incubated in $1 \%$ BSA (Sigma) for $1 \mathrm{~h}$ to block non-specific proteinprotein interactions. IGF2 was stained by using a polyclonal rabbit anti-IGF2 antibody (1:50, Abbiotec, San Diego, CA) for $1 \mathrm{~h}$. IGF1R and IGF2R were stained with polyclonal rabbit anti-IGF1R (1:100; EnoGene Biotech, New York, NY) and polyclonal rabbit anti-IGF2R (1:100,
Bioworld Technology, St. Louis Park, MN), respectively. After several washes with PBS containing 0.1\% BSA (Sigma), the cells were incubated with the secondary antibody, FITC-conjugated goat-anti-rabbit $\operatorname{IgG}(\mathrm{H}+\mathrm{L}) \mathrm{Ab}$ (1:200), for $1 \mathrm{~h}$ at room temperature. Nuclei were visualized by Hoechst 33342 (Wako, Osaka, Japan) staining. Confocal microscopy was performed with a Zeiss LSM 510 microscope (Carl Zeiss, Oberkochen, Germany).

Statistical analysis. Results are presented as the means \pm standard deviation of three independent experiments. Data were statistically analyzed by the Student's $t$ test. $P<0.05$ was regarded as statistically significant.

\section{Results}

We previously found that ameloblastin expression in HAT-7 cells was induced by co-culture with $\mathrm{BCPb} 8$ cells by using the collagen membrane (Matsumoto et al. 2011). We have constructed two co-culture models to investigate the interaction between two different dental cell lines, a rat HAT-7 cell line that originated from dental epithelia, and bovine $\mathrm{BCPb} 8$ cells, derived from cementoblast progenitor cells. One co-culture method (SW) cultured these cells by inoculation of the cell lines onto opposite sides of a collagen film suspended in a cell culture dish. The second, control method, termed "SC" cultured $\mathrm{BCPb} 8$ cells on the culture dish and the HAT-7 cells on a collagen film that faced away from the BCP8 cells on the dish. The results suggested that the molecular weights of substances that pass through the collagen membrane should be less than $12.5 \mathrm{kDa}$. IGFs are one of the factors to induce ameloblastin, whose molecular weight is about $8 \mathrm{kDa}$. IGFs are known to influence ameloblast differentiation and the production of various enamel matrix components (Joseph et al. 1993; Joseph et al. 1994; Joseph et al. 1996; Joseph et al. 1997; Takahashi et al. 1998; Caton et al. 2005). 


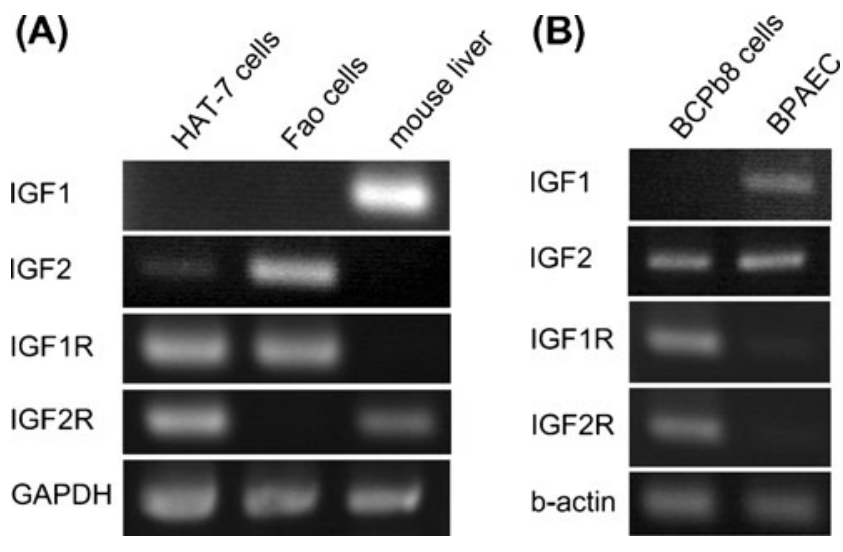

Figure 1. Expression of IGFs and IGFRs in HAT-7 and BCPb8 cells. Electrophoresis of products after RT-PCR amplification with IGF1, IGF2, IGF1R, and IGF2R in HAT-7 ( $A$, lane 1$)$ and $\mathrm{BCPb8}(B$, lane 1$)$ cells. RNA was extracted from HAT-7 cells and $\mathrm{BCPb} 8$ cells cultured on tissue-culture polystyrene dishes. Fao cells ( $B$, lane 2$)$, mouse liver $(A$, lane 3), and BPAEC ( $B$, lane 2) RNA were used as positive controls.

First, we checked the gene expression of the IGF and IGFR family members in $\mathrm{HAT}-7$ and $\mathrm{BCPb} 8$ cells. IGF1R and IGF2R mRNAs were detected in HAT-7 and
$\mathrm{BCPb} 8$ cells by using RT-PCR (Fig. 1). The expression levels of IGF1 and IGF2 in HAT-7 cells were not detected (Fig. 1A). The expression level of IGF1 in BCPb8 cells was lower than in control cells (Fig. 1B). IGF2 mRNA was expressed in $\mathrm{BCPb} 8$ cells at the same level as in BPAEC (Fig. 1B). These results suggest that IGF2 may be important for the interaction between HAT-7 and BCPb8 cells.

The measurement of cell to cell cross-talk at gene expression levels is one method to examine interactions between deferent cell types. However, it is generally difficult determine the gene expression of specific cell types in coculture. To overcome these obstacles, we have used the species-specific primers for different species cells co-culture (Kurosawa et al. 2005). We analyzed the expression level of IGF2 mRNA when BCPb8 cells were co-cultured with HAT7 cells in the SC and SW systems (Fig. 2). The specific rat primers used for this analysis were designed from regions of low homology between rat and bovine cDNA to avoid crossamplification. The IGF2 levels in BCPb8 cells in both coculture systems showed a gradual increase throughout $14 \mathrm{~d}$. In the SW culture, IGF2 expression levels on day 14 were significantly increased by fourfold as compared with day 1
Figure 2. IGF2 mRNA expression of $\mathrm{BCPb} 8$ cells was increased by sandwich co-culture with HAT-7 cells. Quantitative analysis of IGF2 mRNA from $\mathrm{BCPb} 8$ cells in separate co-culture $(A)$ and sandwich co-culture $(B)$ with HAT-7 cells as compared with the mono-culture control. Total RNA was prepared from cells at each designated time point. After synthesizing cDNA from total RNA, gene expression levels were analyzed by real-time PCR with species-specific primers. Data were normalized to $\beta$-actin gene expression levels. IGF2 was detected on co-culture days 1 $(C)$ and $14(D)$. Values are presented as mean \pm standard deviation $(n=3)$. Data were statistically analyzed with the Student's $t$ test $\left({ }^{*} p<0.05\right)$.
(A)

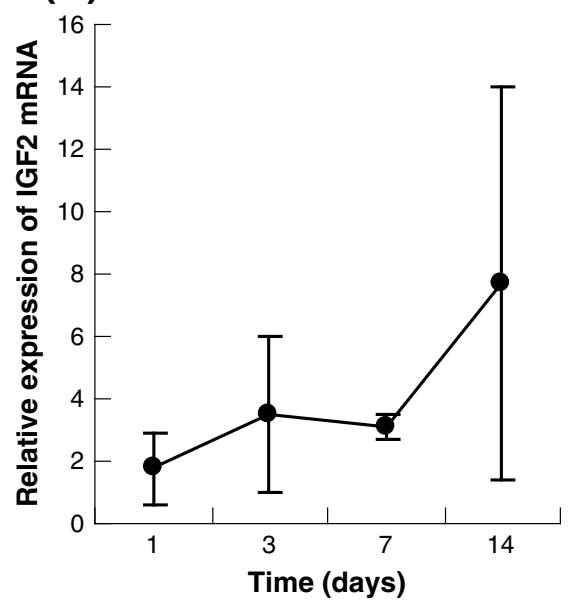

(C)

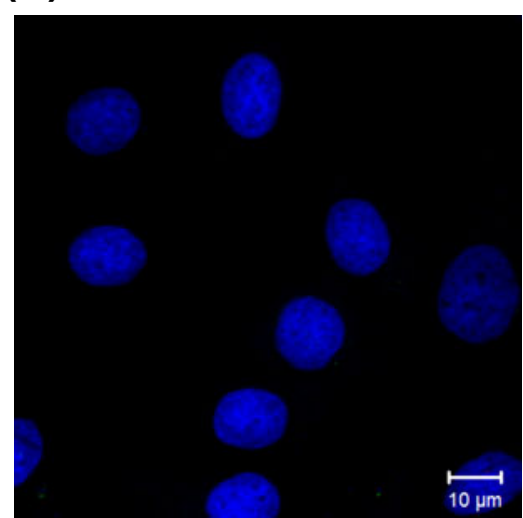

(B)

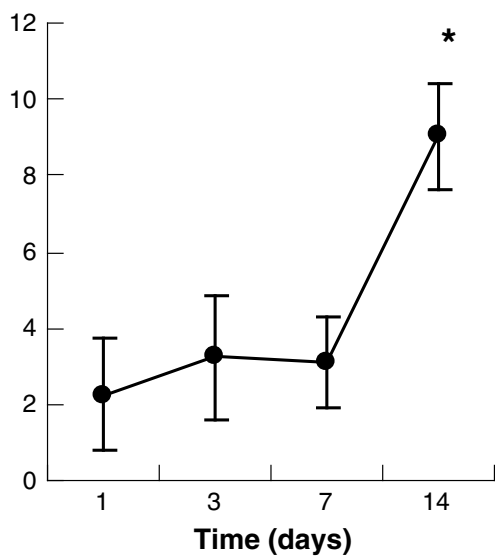

(D)

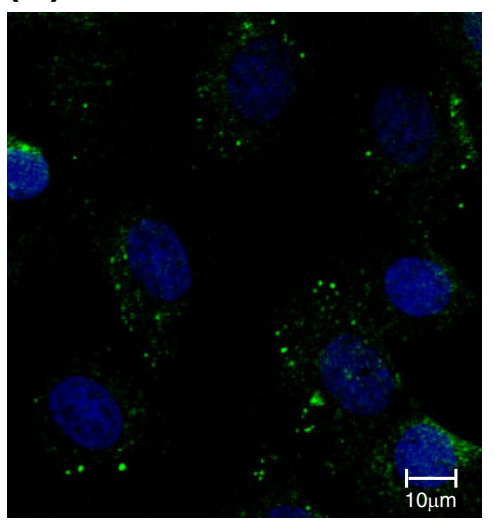


Figure 3. IGF1R and IGF2R mRNA expression of HAT-7 cells by separate and sandwich co-culture with $\mathrm{BCPb} 8$ cells. Quantitative analysis of IGF1R and IGF-2R mRNA expression from HAT-7 cells by separate (left panel) and sandwich (right panel) co-culture with $\mathrm{BCPb} 8$ cells as compared with the mono-culture control. Total RNAs were prepared from cells at days 1 and 14. After synthesizing cDNA from total RNA, gene expression levels were analyzed by real-time PCR with species-specific primers. Data were normalized to corresponding GAPDH gene expression levels. $S W$ sandwich co-culture, $S C$ separate co-culture. IGF2R was not detected in co-culture on days 1 $(C)$ and $14(D)$. Values are presented as mean \pm standard deviation $(n=3)$. Data were statistically analyzed with the Student's $t$ test $\left({ }^{*} p<0.05\right)$.

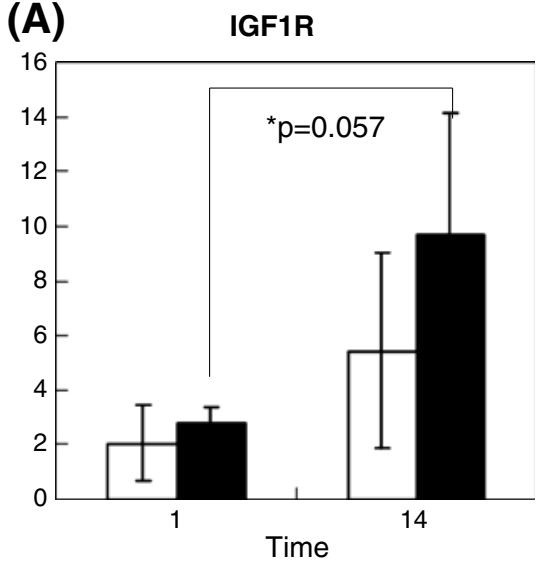

(C)

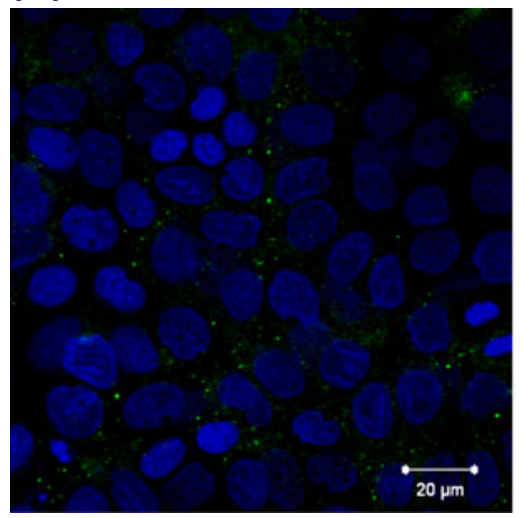

(B) IGF2R

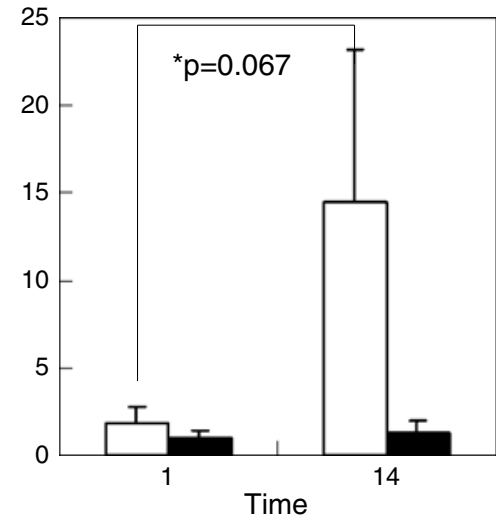

(D)

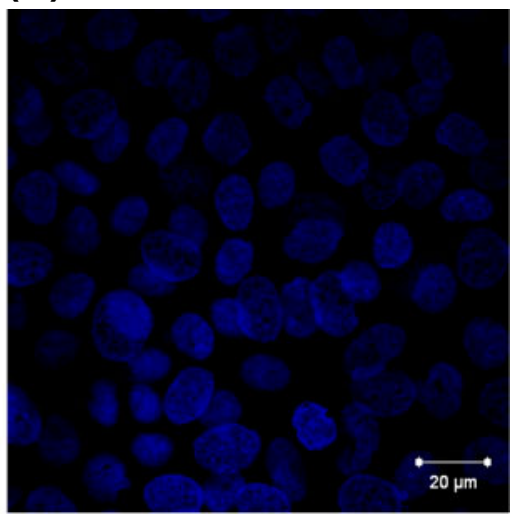

(Fig. 2B). Immunohistochemistry with anti-IGF2 IgG showed that the IGF2 proteins produced in $\mathrm{BCPb} 8$ cells on day 14 were higher than on day 1 (Fig. $2 C, D$ ). These results suggest that soluble factor(s) from HAT-7 cells caused the

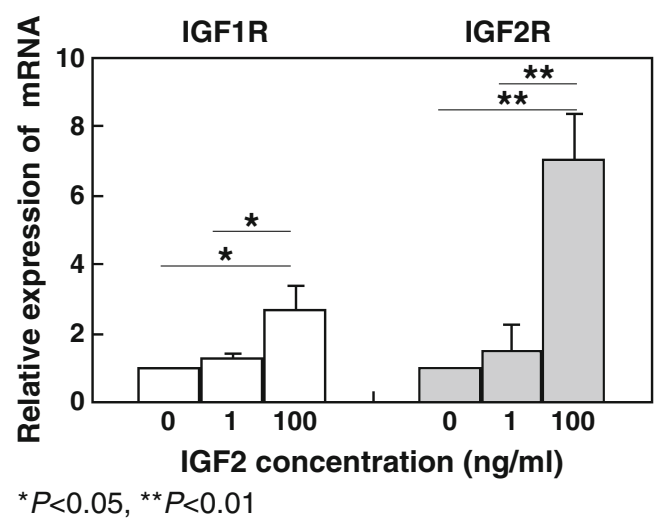

Figure 4. IGF2 induced IGF1R and IGF2R mRNA expression in mono-cultured HAT-7 cells. Quantitative analysis of IGF1R and IGF2R mRNA from mono-cultured HAT-7 cells treated with 1 and $100 \mathrm{ng} / \mathrm{ml}$ of IGF2 as compared with the untreated controls. Total RNA was prepared from cells at each designated time point. After synthesizing cDNA from total RNA, gene expression levels were analyzed by real-time PCR with species-specific primers. Data were normalized to the corresponding GAPDH gene expression levels. Values are presented as mean \pm standard deviation $(n=3)$. Data were statistically analyzed with the Student's $t$ test $\left({ }^{*} p<0.05\right)$. expression of IGF2 mRNA and proteins in $\mathrm{BCPb} 8$ cells via the collagen membrane.

Next, we determined IGFR expression levels on days 1 and 14. IGF2 can bind to IGF1R and IGF2R. IGF2R does not induce intercellular signals because of the degradation of IGF2. IGF1R expression levels on day 14 were increased by up to six times in $\mathrm{SC}$ and ten times by $\mathrm{SW}$ (Fig. 3A). IGF2R mRNA of the SC co-culture dramatically increased during the $14 \mathrm{~d}$. However, IGF2R mRNA of the SW co-culture did not increase during the $14 \mathrm{~d}$ (Fig. 3B). On day 14, IGF1R protein was detected in HAT-7 cells (Fig. 3D), but IGF2R protein was not detected in HAT-7 cells by immunohistochemistry (Fig. 3C). These results show that IGF2 signals were effective in SW as compared with SC.

To understand the effect of IGF2 on tooth development, we added recombinant IGF2 protein to HAT-7 cells on a collagen membrane. We analyzed the expression levels of IGF receptors and ameloblastin, which is a differentiation maker of HAT-7 cells. IGF1R and IGF2R expression levels in HAT-7 cells were increased by the addition of recombinant IGF2 (Fig. 4). The level of IGF1R expression was increased by three times as much as that of no treatment. IGF2R transcripts increased seven times higher than the control level, which is similar to SC co-culture. These results indicate that recombinant IGF2 protein induces IGF1R and IGF2R expression levels in HAT-7 cells on a 
collagen membrane. IGF2R transcripts were not increased in SW (Fig. 3B). Our results did not show how to suppress to induce IGF2R induction in SW. One possible hypothesis for this phenomenon is that other cytokines, which were stabilized and concentrated by collagen membrane, suppress to induce IGF2R induction in SW.

\section{Discussion}

Our system reconstructs part of the tooth development process; therefore, it appears that IGF2 plays a role in tooth differentiation. Previous reports indicate that IGF2 affects enamel differentiation (Caton et al. 2005). However, ameloblastin expression levels barely changed after the addition of IGF2 to HAT-7 cells. IGF2 alone is not enough to induce the differentiation of HAT-7 cells; IGF2 requires collaboration with other molecules, e.g., IGFBPs, the extracellular matrix (ECM), and growth factors. IGFBPs function not only to transport IGFs in biological fluids, therefore prolonging their half-lives, but they also act as modulators of IGF availability and activity (Jones and Clemmons 1995).

Our study showed that IGF2 transcripts increase in $\mathrm{BCPb} 8$ cells only when co-cultured with HAT-7 cells. We also showed that IGF2R levels in HAT-7 cells were inhibited in the SW system. These results indicated that soluble factor(s) from HAT-7 cells induce the expression of IGF2 mRNA and proteins in $\mathrm{BCPb} 8$ cells while $\mathrm{BCPb} 8$ cell-derived factors inhibit the expression level of IGF2R in HAT-7 cells in the SW system. These factors act through the ECM, therefore they also should be smaller than $12.5 \mathrm{kDa}$ to pass through the collagen membrane. Furthermore, our data indicated that the collagen membrane might play a role not only in the accumulation of but also in the stabilization of, soluble factors from $\mathrm{BCPb} 8$ cells.

We believe that it is critical to focus on IGFs to understand the mechanism of the interaction between epithelial and mesenchymal tissue during tooth development.

This study indicates the importance of the interaction between ECM and growth factors, and it may reveal the importance of IGF2R inhibitor for enamel differentiation.

\section{References}

Caton J.; Bringas Jr. P.; Zeichner-David M. IGFs increase enamel formation by inducing expression of enamel mineralizing specific genes. Arch Oral Biol 50: 123-129; 2005.
Denley A.; Cosgrove L. J.; Booker G. W.; Wallace J. C.; Forbes B. E. Molecular interactions of the IGF system. Cytokine Growth Factor Rev 16: 421-439; 2005.

Jernvall J.; Keranen S. V.; Thesleff I. Evolutionary modification of development in mammalian teeth: quantifying gene expression patterns and topography. Proc Natl Acad Sci U S A 97: 14444$14448 ; 2000$.

Jones J. I.; Clemmons D. R. Insulin-like growth factors and their binding proteins: biological actions. Endocr Rev 16: 3-34; 1995.

Joseph B. K.; Savage N. W.; Daley T. J.; Young W. G. In situ hybridization evidence for a paracrine/autocrine role for insulin-like growth factor-I in tooth development. Growth Factors 13: 11-17; 1996.

Joseph B. K.; Savage N. W.; Harbrow D. J.; Young W. G. Nonexpression of insulin-like growth factor-I receptor is associated with apoptosis: an ultrastructural study on rat ameloblasts. Apoptosis 2: 471-477; 1997.

Joseph B. K.; Savage N. W.; Young W. G.; Gupta G. S.; Breier B. H.; Waters M. J. Expression and regulation of insulin-like growth factor-I in the rat incisor. Growth Factors 8: 267-275; 1993.

Joseph B. K.; Savage N. W.; Young W. G.; Waters M. J. Insulin-like growth factor-I receptor in the cell biology of the ameloblast: an immunohistochemical study on the rat incisor. Epithelial Cell Biol 3: 47-53; 1994.

Kawano S.; Morotomi T.; Toyono T.; Nakamura N.; Uchida T.; Ohishi M.; Toyoshima K.; Harada H. Establishment of dental epithelial cell line (HAT-7) and the cell differentiation dependent on Notch signaling pathway. Connect Tissue Res 43: 409412; 2002.

Kurosawa Y.; Taniguchi A.; Okano T. Novel method to examine hepatocyte-specific gene expression in a functional coculture system. Tissue Eng 11: 1650-1657; 2005.

Le Roith D. The insulin-like growth factor system. Exp Diabesity Res 4: 205-212; 2003.

Matsumoto A.; Harada H.; Saito M.; Taniguchi A. Induction of enamel matrix protein expression in an ameloblast cell line cocultured with a mesenchymal cell line in vitro. In Vitro Cell Dev Biol Anim 47: 39-44; 2011.

Petley T.; Graff K.; Jiang W.; Yang H.; Florini J. Variation among cell types in the signaling pathways by which IGF-I stimulates specific cellular responses. Horm Metab Res 31: 70-76; 1999.

Saito M.; Handa K.; Kiyono T.; Hattori S.; Yokoi T.; Tsubakimoto T.; Harada H.; Noguchi; Saito M.; Handa K.; Kiyono T.; Hattori S.; Yokoi T.; Tsubakimoto T.; Harada H.; Noguchi T.; Toyoda M.; Sato $\mathrm{S}$. et al. Immortalization of cementoblast progenitor cells with Bmi-1 and TERT. J Bone Miner Res 20: 50-57; 2005.

Takahashi K.; Yamane A.; Bringas P.; Caton J.; Slavkin H. C.; Zeichner-David M. Induction of amelogenin and ameloblastin by insulin and insulin-like growth factors (IGF-I and IGF-II) during embryonic mouse tooth development in vitro. Connect Tissue Res 38: 269-278; 1998. discussion 295-303.

Thesleff I. Epithelial-mesenchymal signalling regulating tooth morphogenesis. J Cell Sci 116: 1647-1648; 2003.

Thesleff I.; Mikkola M. The role of growth factors in tooth development. Int Rev Cytol 217: 93-135; 2002.

Thesleff I.; Sharpe P. Signalling networks regulating dental development. Mech Dev 67: 111-123; 1997.

Werner H.; Katz J. The emerging role of the insulin-like growth factors in oral biology. $J$ Dent Res 83: 832-836; 2004. 